\title{
Globe
}

Revue internationale d'études québécoises

\section{Parutions récentes en études québécoises}

\section{Michel Lacroix}

Volume 8, numéro 1, 2005

URI : https://id.erudit.org/iderudit/1000905ar

DOI : https://doi.org/10.7202/1000905ar

Aller au sommaire du numéro

Éditeur(s)

Globe, Revue internationale d'études québécoises

ISSN

1481-5869 (imprimé)

1923-8231 (numérique)

Découvrir la revue

Citer ce document

Lacroix, M. (2005). Parutions récentes en études québécoises. Globe, 8(1),

245-249. https://doi.org/10.7202/1000905ar d'utilisation que vous pouvez consulter en ligne.

https://apropos.erudit.org/fr/usagers/politique-dutilisation/ 


\section{Parutions récentes en études québécoises}

$1^{\text {er }}$ janvier 2005 au 30 juin 2005

Nos lecteurs sont invités à nous faire part des parutions récentes en études québécoises publiées à l'étranger.

Robert Aird, André Patry et la présence du Québec dans le monde, Montréal, VLB, coll. *Études québécoises ", 2005, ISBN 2-89005-908-1 [biographie d'un diplomate québécois].

Élyse Arbic [éd.], Le Québec en 2025. Visions de la jeune génération, Rosemère, J. Cornu, coll. "Jeune plume ", 2005, ISBN 2-922976-07-6 [écrits de jeunes québécois].

Gérald Arbour, et autres [éd.], Les ponts couverts au Québec, Sainte-Foy, Publications du Québec, 2005, ISBN 2-551-19636-1 [élaboré à l'initiative et sous la supervision du ministère des Transports du Québec].

Rose Marie Arbour, 9 vues. Euvre gravée de Louise Masson [avec récit poétique de Daniel Danis], Montréal, Éditions du Passage, 2005, ISBN 2922892-11-5.

Paul Aubin et Michel Simard, Les manuels scolaires dans la correspondance du Département de l'industrie publique, 1900-1920. Inventaire, Sherbrooke, Ex Libris, coll. • Cahiers du GRELQ •, nº 9, 2005.

Michel Lacroix [éd.], - Parutions récentes en études québécoises •, Globe. Revue internationale d'études québécoises, vol. 8, $\mathrm{n}^{\circ} 1,2005$. 


\section{REVUE INTERNATIONALE D'ÉTUDES QUÉBÉCOISES}

Antoine Baby, Pédagogie des poqués, Sainte-Foy, Presses de l'Université du Québec, coll. "Éducation intervention ", 2005, ISBN 2-7605-1340-8 [sur le réseau québécois des CFER et la formation professionnelle des jeunes].

Pascale Beaudet, Josée Fafard. Les pieds sur terre lavant-propos de France Gascon], Joliette, Musée d'art de Joliette, 2005, ISBN 2-921801-32-9.

Marilou Brousseau, Le jeune de cour. André Lejeune. Une voix vibrante du Québec, Saint-Hubert, Éditions Un Monde différent, coll. * UMD , 2005, ISBN 2-89225-585-6.

Jacques Castonguay, Comment a-t-on fermé le Collège militaire de SaintJean ?, Montréal, Art global, 2005, ISBN 2-920718-97-5.

Citoyenneté et immigration Canada, Les nouveaux immigrants de la région métropolitaine de Montréal. Profil comparatif établi d'après le recensement de 2001, Ottawa, Citoyenneté et immigration Canada, 2005, ISBN 0-662-78741-2.

John B. Claxton, Studies on the Quebec Law of Trust, Toronto, Thomson Carswell, 2005, ISBN 0-459-24173-7.

Bernard Cliche, et autres, Le barcèlement psycbologique et les lésions psychologiques, Cowansville, Éditions Y. Blais, 2005, ISBN 2-89451-813-7 [droit du travail québécois].

Anne-Marie Cloutier, Le dépit amoureux. Têmoignages sur la critique théâtrale au Québec, Saint-Laurent, Fides, 2005, ISBN 2-7621-2652-5.

Marcel Dubé, André Pitre, Montréal, Art global, 2005, ISBN 2-92071896-7 [entretiens avec un peintre québécois].

Jane Everett [éd.], In translation. The Gabrielle Roy-Joyce Marshall correspondence, Toronto, University of Toronto Press, 2005, ISBN-0-80203908-1.

Magdalena Fahrni, Housebold Politics. Montreal Families and Postwar Reconstruction, Toronto, University of Toronto Press, coll. "Studies in gender and history ", 2005, ISBN 0-8020-3849-2 [relié], ISBN 0-80204888-9 [poche]. 


\section{PARUTIONS RÉCENTES EN ÉTUDES QUÉBÉCOISES}

Michèle Febvre et Guylaine Massoutre, Anatomie du vertige. Ginette Laurin. 20 ans de création, avant-propos de Denis Marleau, Montréal, Heures bleues, 2005, ISBN 2-922265-28-5.

Robert Filion, Une saison chez Camille Laurin. Carnet d'un compagnon de route, Montréal, I. Quentin, coll. *Groupie ", 2005, ISBN 2-92241751-4.

Louise H. Forsyth [éd.], Nicole Brossard. Essays on ber Works, Toronto, Guernica, coll. - Writers series ", 2005, ISBN 1-55071-233-0.

Ludovic Fouquet, Robert Lepage, l'borizon en images, Québec, L'Instant même, coll. "L'instant scène *, 2005, ISBN 2-89502-210-0.

François Gendron, L'affaire des trâ̂tres. Essai sur la liberté de parole en matière politique, Montréal, Wilson \& Lafleur, coll. "Paroles d'avocats ", 2005, ISBN 2-89127-714-7.

Guy Giguère, 'Les brebis égarées, Outremont, Stanké, 2005, ISBN-2-76040953-8 [sur les mœurs et coutumes du Québec avant 1763].

Danny Kucharsky, Sacred ground on de la Savane. Montreal's Baron de Hirscb Cemetery, Montréal, Véhicule Press, 2005, ISBN 1-55065-196-X.

Jeannine Laurent et Jacques Saint-Pierre, Policiers et pompiers en devoir, 1851-1977, Sainte-Foy, Publications du Québec, coll. "Aux limites de la mémoire *, 2005, ISBN 2-551-19689-2.

Georges Leroux et Pierre Ouellet [éd.], L'engagement de la parole. Politique du poème, Montréal, VLB, coll. "Le soi et l'autre ", 2005, ISBN 289005-915-4.

Laurent Mailhot, Les plaisirs de la prose, Montréal, Presses de l'Université de Montréal, 2005, ISBN 2-7606-1987-7.

Amélie Nadeau, Une passerelle entre le réel et l'imaginaire. L'univers musical dans les Chroniques du plateau Mont-Royal et L'oratorio de Noël de Göran Tunström, Montréal, Imaginaire/Nord, Laboratoire international d'étude multidisciplinaire comparée des représentations du Nord, coll. * Droit au pôle ^, 2005, ISBN 2-923385-01-2. 


\section{REVUE INTERNATIONALE D'ÉTUDES QUÉBÉCOISES}

Louise Otis, La transformation de notre rapport au droit par la médiation judiciaire, Montréal, Éditions Thémis, coll. "Conférence AlbertMayrand n, 2005, ISBN 2-89400-205-X.

Jean-Louis Roy, Montréal. Ville nouvelle, ville plurielle. La renaissance inachevée, Montréal, Hurtubise HMH, 2005, ISBN 2-89428-819-0.

Michel Venne [éd.], 100 idées citoyennes pour un Québec en santé, SaintLaurent, Fides, 2005, ISBN 2-7621-2642-8.

\section{Traductions}

Florence Bolté, Snow castles, avec illustrations de Mentalo, Itraduction de Châteaux de neige par Philip Lee], Mont-Saint-Hilaire, Pirouli, coll. " Aïxa *, 2005, ISBN 2-922754-05-7.

Florence Bolté, Castelli di neve, avec illustrations de Mentalo, Itraduction de Châteaux de neige par Evelina Mosetti], Mont-Saint-Hilaire, Pirouli, coll. "Aïxa ", 2005, ISBN 2-922754-07-3.

Nicole Brossard, Yesterday, at the Hotel Clarendon, Itraduction de Hier par Susanne de Lotbinière-Harwood], Toronto, Coach House Books, 2005, ISBN 1-55245-150-X.

Normand Chaurette, The concise Köchel, Itraduction de Le petit Köchel par Linda Gaboriau], Vancouver, Talonbooks, 2005, ISBN-0-88922-518-4.

Francine D'Amour, Return from Africa. A novel, Itraduction de Le retour d'Afrique par Wayne Grady], Vancouver, Douglas \& McIntyre, 2005, ISBN 1-55365-098-0.

Daniel Danis, In the eyes of stone dogs, [traduction de Langue-à-langue des chiens de roche par Linda Gaboriau], Vancouver, Talonbooks, 2005, ISBN 0-88922-519-2.

Martine Desjardins, All that glitters, [traduction de Élu du basard par Fred A. Reed et David Homell, Vancouver, Talonbooks, 2005, ISBN 0-88922520-6. 


\section{PARUTIONS RÉCENTES EN ÉTUDES QUÉBÉCOISES}

Jean-Claude Dubé, The chevalier de Montmagny, 1601-1657. First governor of New France, [traduction de Le chevalier de Montmagny par Elizabeth Rapleyl, Ottawa, University of Ottawa Press, coll. a Amérique française *, 2005, ISBN 0-7766-3028-8 [relié], ISBN 0-7766-0559-3 [poche].

Sinclair Dumontais, The parachute. A sadly funny novel about the consumption of consumers, Itraduction de Le paracbute de Socrate par Patricia Claxton], Toronto, Key Porter Books, 2005, ISBN 1-55263-734-4.

François Gravel, Adieu, Betty Crocker, Itraduction de Adieu, Betty Crocker par Sheila Fischman], Toronto, Cormorant Books, 2005, ISBN 1896951-60-0.

Andrée Lévesque [éd.], Madeleine Parent, activist, [traduction de Madeleine Parent, militante par Andrée Lévesque], Toronto, Sumach Press, 2005, ISBN 1-894549-46-5.

David Solway, Demilunes. Little windows on Quebec, [traduction de Demilunes par David Solway], Victoria, Frog Hollow Press, coll. "Contemporary Canadian poets •, 2005 [anthologie de poésie].

France Théoret, Girls closed in, \{traduction de Huis clos entre jeunes filles par Luise von Flotow], Toronto, Guernica, coll. "Prose series ", 2005, ISBN 1-55071-206-3.

Larry Tremblay, The bicycle eater, [traduction de Le mangeur de bicyclette par Sheila Fischman], Vancouver, Talonbooks, 2005, ISBN 0-88922-528-1.

Michel Tremblay, The driving force, [traduction de L'impératif présent par Linda Gaboriau], Vancouver, Talonbooks, 2005, ISBN 0-88922-530-3. 